\title{
Gass's Atlas of Macular Diseases Fifth Edition: A Agarwal, A Singh 2012. Elsevier. ISBN 978-1-4377-1580-4
}

\author{
Shimon Rumelt
}

Received: 5 November 2012 / Accepted: 7 November 2012 / Published online: 29 November 2012

(C) Springer-Verlag Berlin Heidelberg 2012

This updated comprehensive atlas is justly considered to be the bible of macular diseases. It includes pure macular diseases as well as macular diseases affecting the retinal periphery, in addition to optic nerve head diseases that mimic macular diseases. The presentation of the diseases is according to their etiology, without subdivision to the involved tissue (retina, retinal pigment epithelium, and choroid). Since these are macular diseases, almost all affect either visual acuity, visual field, color discrimination, or contrast sensitivity, while a very few may have a subtle impact on any on these parameters. The text comprises 15 sections that include all the macular diseases, including rare and newly described diseases. The first two chapters describe the clinical, anatomical, and physiological features of the normal macula, rather than the diseases, and finally optic nerve head diseases are described. The tumors are described in three chapters (RPE, retina, and choroid). The description of each disease includes the medical history, ocular examination, pathogenesis, and differential diagnosis. Each chapter is accompanied by an extensive reference list.

The new edition includes new entities such as EpsteinBarr and West Nile viral retinitis, acute idiopathic maculopathy, persistent relentless placoid pigment epitheliopathy, Bothnia dystrophy, and Newfoundland rod-cone dystrophy as well as ocular coherence tomography (OCT) images.
Nevertheless, there is no replacement for fluorescein angiography. Other relevant images such as ultrasound and computerized tomography are provided. Non-retinal manifestations are described without photos. The online version includes the 3D images that appeared in print in the past. The description of the images is as case presentations.

The text is accompanied by superb clinical, schematic, and histologic as well as imaging photographs. (fluorescein angiography, indocyanine green, and optical coherence tomography). Some of the clinical images contain arrows to point out the pathology, and hopefully in the next updates, arrows and index to the other figures and additional imaging images for those entities which lack them would be added.

This book was a lifetime project for Dr. Donald M. Gass and now his successor Dr. Anita Agarwal and her co-author Dr. Arun Singh. It is hoped that the project of updating and expanding this book will be continued for the benefit of the entire ophthalmic community and its patients worldwide.

Hopefully, the reader will have an indefinite access to the online version of the book, the authors will add more OCT images for the diseases that do not include them, and will mark the pathologic findings on the images in the next editions.

Conflict of interest None

S. Rumelt $(\bowtie)$

Western Galilee Nahariya Medical Center, Nahariya, Israel

e-mail: rumelt@zahav.net.il 